\title{
Digital image processing: a useful tool in the analysis of lung injuries caused by chronic inhalation of agricultural herbicides
}

\author{
Claudio Pedrassoli Junior ${ }^{1}$. Gabriela Vidotto Cavallieri ${ }^{2}$. Francisco Assis da Silva ${ }^{3}$ - Geovana Letícia Fernandes ${ }^{4}$. \\ Gisele Alborghetti Nai ${ }^{4}$. Ana Karina Marques Salge ${ }^{5}$. Josiano Guilherme Puhle ${ }^{6}$. Debora Tavares de Resende e \\ Silva ${ }^{7}$. Danillo Roberto Pereira ${ }^{8} \cdot$ Fabíola de Azevedo Mello $^{9} \cdot$ Ana Paula Alves Favareto ${ }^{10} \cdot$ Renata Calciolari Rossi $^{11}$
}

Received: 11 March 2021 / Accepted: 31 May 2021 / Published online: 7 June 2021

(C) The Author(s), under exclusive licence to Springer-Verlag GmbH Germany, part of Springer Nature 2021

\begin{abstract}
The herbicide 2,4-dichlorophenoxyacetic acid (2,4-D) is widely used in agriculture to control various weeds. The objective of this study was to use the digital image processing method to identify alveolar lesions in the lungs of rats submitted to chronic 2,4dichlorophenoxyacetic acid (2,4-D) inhalation exposure. We used forty adult male Wistar rats. The rats were divided into four groups: control group (CG), low concentration group (LCG), medium concentration group (MCG), and high concentration group (HCG). In a 6-month exposure period, we used two boxes connected to ultrasonic nebulizers for herbicide spraying. After this period, the rats were euthanized for the collection and study of lung tissue. For each image, counts of injuries and blisters were performed automatically using a methodology based on digital image processing techniques. For analysis of the results, an electronic database $\left(\right.$ Excel $\left.{ }^{\circledR}\right)$ was created. We used the Pearson method for correlation analysis; values of $p<0.05$ were considered significant. In the evaluation of healthy alveoli, we recorded positive and significant correlations between analysis from a pathologist and computational analysis. In the evaluation of injured alveoli, we recorded a positive but non-significant correlation between analysis from a pathologist and computational analysis. These results show the effectiveness of digital image processing when evaluating alveolar integrity.
\end{abstract}

Keywords Digital image processing $\cdot$ Herbicide $\cdot 2,4$-Dichlorophenoxyacetic acid $\cdot$ Inflammation $\cdot$ Alveolar lesions $\cdot$ Lung

\section{Introduction}

The success of plantations often depends on weed control actions, such as pesticide use. Since 2008, Brazil has been the global leader in the consumption of crop protection

Responsible Editor: Lotfi Aleya

Renata Calciolari Rossi

renatacalciolari@terra.com.br

1 Environmental and Regional Development, Oeste Paulista University, Presidente Prudente, SP, Brazil

2 Health Sciences, Oeste Paulista University, Presidente Prudente, SP, Brazil

3 Computer Engineering, University of São Paulo, São Carlos, SP, Brazil

4 Oeste Paulista University, Presidente Prudente, SP, Brazil

5 Federal University of Goiás, Goiânia, GO, Brazil

6 Federal University Fronteira do Sul, Chapecó, SC, Brazil products. The abusive use of pesticides is directly related to numerous health problems and affects workers in the agribusiness production chain, residents of areas near production centers, and consumers who ingest derivatives of herbicide-aided crops (Abrasco 2016; Gomes 2002).

7 Health Sciences, Federal University Fronteira do Sul, Chapecó, SC, Brazil

8 Environmental and Regional Development, Presidente Prudente, SP, Brazil

9 Animal Science, Oeste Paulista University, Presidente Prudente, SP, Brazil

10

Environmental and Regional Development, Oeste Paulista University, Presidente Prudente, SP, Brazil

11 Environmental and Regional Development, Oeste Paulista University, Km 572, SP-270 - Bairro Limoeiro, Pres. Prudente, SP 19026-310, Brazil 
Inhaled particles can settle in the airways, where they interfere with lung-cleaning mechanisms, or deep in lungs. In cases of long-term exposure, these effects can eventually contribute to the development of chronic obstructive pulmonary disease (COPD), emphysema, and lung cancer. Lungs serve as gateways to systemic circulation. Thus, chronic inhalation of gases and vapors may cause systemic damage to various organs and systems, including the liver and bone marrow (Firetti et al. 2012).

The health and computing fields have been collaborating to improve parameters related to medical diagnosis. Digital image processing is an example of these efforts; it uses the extraction of patterns from a set of samples to acquire knowledge automatically (Pereira and Artificial intelligence myth and science 2005).

Digital image processing methods can be divided into two basic categories: supervised and unsupervised learning. Supervised learning methods pre-classify a sample set (training set) to extract sample patterns, in which each example used for training is labeled with the value of interest. A supervised learning algorithm searches for patterns among the value labels, while using all relevant information. Such methods need to be classified by a specialist (Koerich 2012).

When using supervised learning methods, the measure of accuracy must be estimated after the training session. This occurs through a set of tests or validations, in which samples are labeled ${ }^{5}$.

Using this method, each sample is classified, and the predicted label is compared with the desired one. This requires an adjustment to make the obtained label equal to the real label of the sample; otherwise, a classification error appears. Through this criterion, the accuracy percentage of the digital image processing method can be calculated, which is then used as an accuracy metric to represent the precision of the classifier (Coppin 2010).

The performance and accuracy of most digital image processing methods are greatly influenced by internal parameters of the algorithms and selection of the characteristics of the data sets for the classification process (Coppin 2010). The findings of this study can be used to make companies more aware of health risks and initiate discussions on sustainable projects that improve health and quality of life.

This study aimed to use the digital image processing method to identify alveolar lesions in the lungs of rats submitted to chronic 2,4-D inhalation exposure.

\section{Materials and methods}

\section{Animals and experimentation environment}

The experimental protocol was approved by the Ethics Committee for Use of Animals (CEUA) at UNOESTE
(Protocol - 3761) and was carried out in accordance with the Guide for the Care and Use of Laboratory Animals of the National Institute of Health (Committee for Update of the guide for the care and use of the laboratory animals 2011).

Adult male Wistar rats $(200-250 \mathrm{~g}, \mathrm{n}=40)$ were used, with an average age of $60 \pm 5$ days. They were placed in collective plastic cages ( 5 rats per cage) that measured $30 \times 16 \times 19 \mathrm{~cm}$. The rats were kept in a temperature-controlled room $(22 \pm$ $2^{\circ} \mathrm{C}, 50-60 \%$ relative humidity) with a 12 -h light/dark cycle (7 am to $7 \mathrm{pm}$, light period; $7 \mathrm{pm}$ to $7 \mathrm{am}$, dark period). Throughout the experimental period, the rats were exposed to the herbicide at the same time of day. The rats received filtered water and commercial rat feed (Supralab®, Alisul, Brazil) ad libitum. All rats from each specific group were exposed to the herbicide at the same time.

\section{2,4-D herbicide exposure protocol}

We used a commercial formulation of (2,4-dichlorophenoxy) acetic acid (2,4-D; Nortox S.A., Arapongas, Paraná, Brazil, registered in the Ministry of Agriculture, Livestock and Food Supply \# 03009). The formulation constituted of $806 \mathrm{~g} / \mathrm{L}$ of dimethylamine salt of (2,4-dichlorophenoxy) acetic acid (2,4D), $670 \mathrm{~g} / \mathrm{L}$ of $2,4-\mathrm{D}$ acid equivalent, and $424 \mathrm{~g} / \mathrm{L}$ of inert ingredients. The 2,4-D formulation was diluted in $0.9 \%$ sodium chloride.

Rats were randomly assigned to four experimental groups ( $\mathrm{n}$ $=10$ (group), as shown in Table 1 . Considering that the rats had the same characteristics (e.g., sex, approximate weight), we randomly divided the rats upon their arrival from the central vivarium by randomly distributing their cages to each exposure group. In the animal experimentation laboratory, the rats were identified by Arabic numbers written on their tails.

Rats from different groups were exposed for five consecutive days per week in a 180-day period. The rats were exposed with the concentration for their specific experimental group (Table 1) (de Mello et al. 2018; de Mello 2020).

Exposure occurred via inhalation in plastic boxes $(32 \times 24 \times 32 \mathrm{~cm})$ that were connected to an ultrasonic nebulizer (Pulmosonic Star ${ }^{\circledR}$, Brazil). Daily exposure periods lasted approximately $15 \mathrm{~min}$; this is the required time for nebulization of the entire solution (de Mello et al. 2018; de Mello 2020).

The 2,4-D concentrations for each experimental group were chosen in accordance with standard product application and agronomic prescription to different cultures. For better interpretation and comparison of results in the literature, 2,4$\mathrm{D}$ concentrations used in agriculture (grams of active ingredient per hectare - g a.i. $\mathrm{ha}^{-1}$ ) were adjusted to the box dimensions and converted to the units of $\mathrm{mg} / \mathrm{m}^{-3}$ and $\mathrm{ppm}$ (de Mello et al. 2018; de Mello 2020).

All rats were exposed to the herbicide for 6 months. They were then euthanized after this period. Anesthesia and euthanasia were performed by sodium thiopental (Syntec, USA) 
Table 1 Experimental groups and 2,4-D exposure concentrations in different units

\begin{tabular}{lllll}
\hline Experimental group & Group acronym & \multicolumn{2}{l}{ Concentration of 2,4-D herbicide } \\
\cline { 4 - 5 } & & $\mathrm{g}$ a.i. ha & $\mathrm{mg} \mathrm{m}^{-3}$ & $\mathrm{ppm}$ \\
\hline Control group & $\mathrm{CG}$ & $0^{*}$ & $0^{*}$ & $0^{*}$ \\
Low concentration group & $\mathrm{LCG}$ & $3.71 \times 10^{-3}$ & 187.17 & 20.69 \\
Medium concentration group & MCG & $6.19 \times 10^{-3}$ & 313.31 & 34.63 \\
High concentration group & HCG & $9.28 \times 10^{-3}$ & 467.93 & 51.66 \\
\hline
\end{tabular}

*Only vehicle: solution of $0.9 \%$ sodium chloride with doses of $100 \mathrm{mg} / \mathrm{kg}$ of weight administered in the peritoneal cavity. Indications of death were reflex loss and absence of respiratory movements and heartbeat.

g.i.a/ha $=$ grams of active ingredient per hectare, considering environmentally relevant concentrations, according to product application in agriculture

$\mathrm{ppm}=$ parts per million

\section{Collection of lung material}

After euthanasia, autopsies were performed to remove lungs for further analysis. For the maintenance of lung morphological architecture and constant pressure $(20 \mathrm{cmH} 2 \mathrm{O})$, we used $10 \%$ buffered formalin in the trachea region. For processing and paraffin block production, we collected lung tissue at random from central and peripheral areas of the lungs of each rat (PARKINSON et al. 2011).

Sample sections of $5 \mu \mathrm{m}$ were stained with hematoxylin eosin for selection of the most appropriate airways for pulmonary epithelium evaluation. We selected areas with preserved histological architecture.

\section{Analysis of lung cell images}

Pathological analyzes were performed by a researcher with experience in pathology. We considered alveoli with similar architectural patterns as healthy. Images classified as "altered" were submitted to the machine-learning process. Classifications were based on specifications used by Ruppert et al. (2012), which considered alveolar damage as the following: involvement and uniform temporal appearance of alveolar collapse; hyaline membranes; obliterative fibrosis; formation of neo-septa and moderately organized fibrosis; intraalveolar hemorrhage characterized by the presence of blood in alveolar spaces; enlarged and swollen alveolar septa, usually combined with mononuclear inflammatory infiltrates.

Normal and injured cells in each image were counted automatically using a methodology based on digital image processing techniques. The images were pre-processed to segment each cell. The segmented cells were then classified as normal or injured, according to metrics obtained from cell images and empirically defined parameters (later described in this section). Figure 1 shows an example of an original image of lung cells with a region marked by two dashed lines in the upper left corner. This demarcated region was cut out from this specific image in order to explain the methodology adopted for pre-processing and cell segmentation. In the image selection process for machine learning, the evaluator who selected the images characterized as "normal" was blinded; the images were previously given random numbers by other researchers.

Each lung cell image (Fig. 2, (a) example of the cropped image of the region marked in Fig. 1) was converted from RGB (red, green, blue) color space of the original image to HSV (hue, saturation, value) color space (Fig. 2) (b). Hue defines the color component. Saturation defines color purity. Value (value/brightness) defines the amount of light (Gonzalez and Woods 2010). In the sequence, an Otsu (1979) threshold was applied only to the " $\mathrm{H}$ " channel of Fig. 2 (b), which generated Fig. 2 (c) image. This image contains a significant highlighted (in red) cell region.

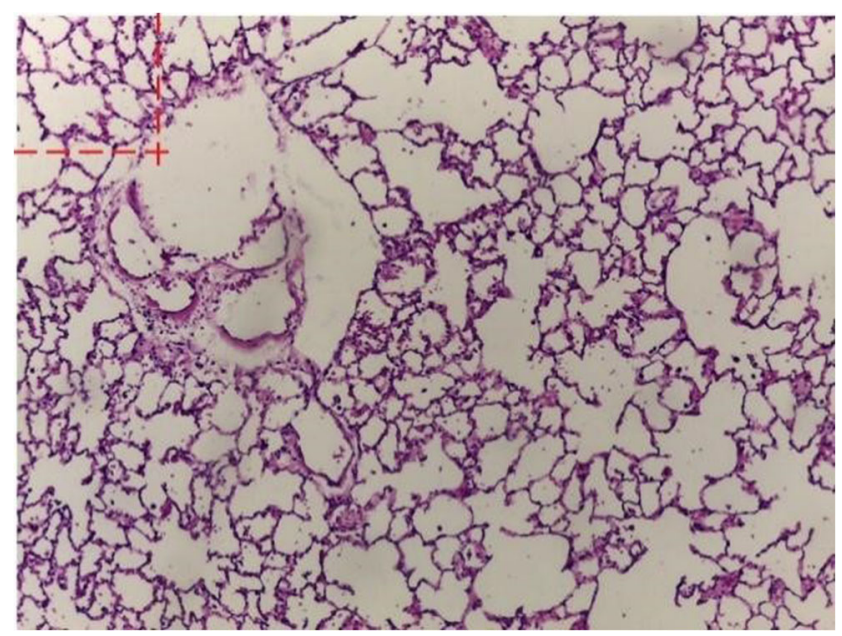

Fig. 1 Original image of a slide of lung cells. The two dashed lines in the upper left corner indicate the region cut out from the image 

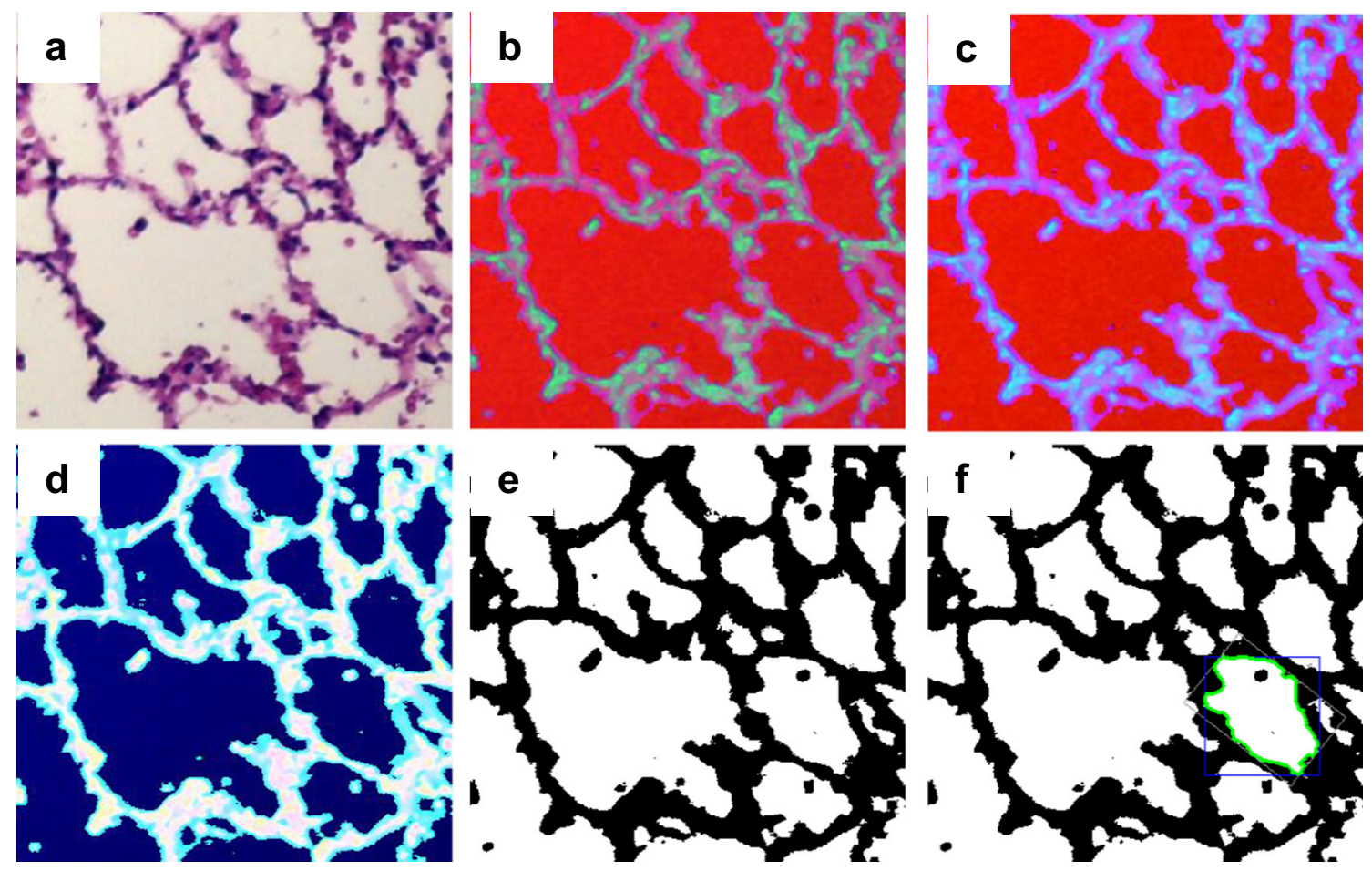

Fig. 2 a Cut out of the original image (Fig. 1). b Image (a) converted from the RGB color space to $\mathrm{HSV}^{7}$. c Image (b) after applying an $\mathrm{Otsu}^{8}$ threshold to channel "H." d Image (c) converted from the YUV ${ }^{9}$ color

For greater cell enhancement, we also used the YUV color space in Fig. 2 (c) image. YUV color space (Y is gray scale or brightness information, $\mathrm{U}$ and $\mathrm{V}$ are the chromaticity or color information in the components) is different from RGB, as it works with separate light and color components (Russ 1999). The image was then converted from the YUV color space to RGB, which resulted in Fig. 2 (d) image. This image shows the cells changing as they move away from the edges.

In addition, we converted Fig. 2 (d) image from the RGB color space to the $\mathrm{HSV}^{7}$ color space. Of the three channels, we only used the "S" channel. We applied channel "S" (a binary threshold) to this image to generate Fig. 2 (e) image. This provides a binary image of the cells separated from the edges without damaging the contour. This process was necessary, as the images of the original cells varied in illumination and were often unclear.

Figure 2 (f) image shows a cell that was segmented by a region-filling algorithm (Gonzalez and Woods 2010). We demarcated the cell by a perimeter (a rectangle surrounding the cell) and calculated the minimum rectangle.

The binary image demonstrated in Fig. 2 (f) (shown with more detail in Fig. 3) contains a seeded cell. It serves as an example of the metrics used for classification of each cell. These metrics include the following:

- cell area (count of the interior pixels of the segmented cell region) calculated using the first region-filling algorithm space to RGB. e Image (d) converted from the RGB color space to HSV (Gonzalez and Woods 2010) and with application of a binary threshold in the " $\mathrm{S}$ " channel, resulting in a binary image. $\mathbf{f}$ Demarcated cell

- cell contour points (perimeter) using the border following algorithm (Suzuki and Abe 1985)

- rectangle surrounding the cell from the Cartesian planes

- minimal rectangle surrounding the segmented cell (possibly rotated) using the contour points obtained by the border following algorithm.

- minimum width using the minimum rectangle calculated from the following expressions:

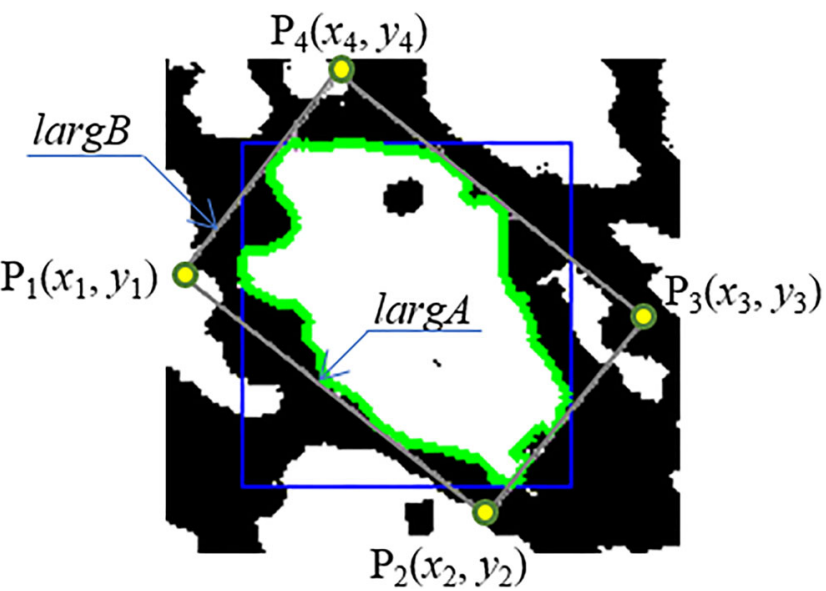

Fig. 3 Magnified detail of a segmented cell. The green line around the cell represents the perimeter. The blue lines represent the rectangle surrounding the cell. The points $\mathrm{P} 1, \mathrm{P} 2, \mathrm{P} 3$, and $\mathrm{P} 4$ represent the coordinates of the calculated minimum rectangle (gray lines) 
The classification of cells as normal and injured was carried out with regard to rules and minimum/maximum parameters. These parameters were empirically attributed to the metrics defined and obtained from the segmented cells of the images. We excluded cells with the following attributes: cells found at the edge of the images, cells less than $43 \times 43$, cells with a total size less than 725 , cells with perimeters less than 175 , and cells with widths less than 37 . From the cells that remained after the previously mentioned exclusions, we used cells with the following attributes: areas less than or equal to a maximum area of 20,500 , perimeters less than or equal to a maximum perimeter of 1250 , sizes less than or equal to a maximum rectangle of $490 \times 490$.

\section{Statistical analysis}

An electronic database (Excel@) was created for the analysis of the results. Pearson's correlation was performed for correlation analyzes, as the samples were normal and homogeneous (Kolmogorov-Smirnov normality test). Values of $p<0.05$ were considered significant.

To determine the minimum sample size for comparison between scores of the eight groups, we calculated the samples using the "pwr" packet (available in Program R) and performed variance analysis (CHAMPELY 2016; R DEVELOPMENT CORE TEAM 2019). After obtaining the minimum number of elements per sample, $15 \%$ more elements were added to each sample for adaption to possible non-parametric distribution (Beaver and Lehmann 2006).

The following parameters were used:

a) Test power $=80 \%$

b) Significance level $=5 \%$

c) Number of groups for comparison $=8$

d) Effect size (Cohen's D) $=0 / 50$

Based on these data, we concluded that at least 12 elements were needed per group. Thus, the value of the sample calculation was $10 \%$.

\section{Results}

We analyzed fragments of lungs from 40 rats that were subjected to chronic 2,4-D exposure. No rats died in the experimental phase of this study. In addition, no complications occurred that would make this study unfeasible. In the evaluation of healthy alveoli, we found positive $\left(r^{2}=0.9234\right)$ and significant $(\mathrm{p}<0.001)$ correlations between analysis from an observing pathologist and computational analysis (Fig. 4).

Our study examined damaged alveoli with areas of inflammatory infiltrates. With damaged alveoli, we recorded a

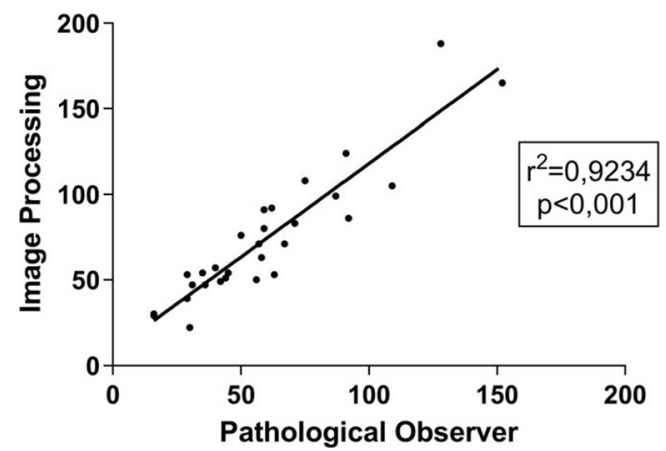

Fig. 4 In healthy alveoli, correlation between analysis from the observing pathologist and computational analysis

positive $\left(\mathrm{r}^{2}=0.2130\right)$ and non-significant $(\mathrm{p}=0.2584)$ correlation between analysis from an observing pathologist and computational analysis (Fig. 5).

\section{Discussion}

Our study demonstrates the effectiveness of digital image processing to identify healthy alveoli, as we recorded positive and significant correlations between pathologist and computational analyses. But different characteristics of the alveolar injuries caused by 2,4-D exposure possibly interfered in the ability to classify damaged alveoli.

Several studies in the literature have examined lung lesion classification. Distinguishing injured lung tissue from uninjured (or healthy) tissue was the subject of a study by Sluimer et al.; they used digital image processing with 657 regions of interest from 116 data sets to distinguish injured from uninjured tissue (Sluimer et al. 2003).

A study by Mansoor et al. (2017) adapted the imaging algorithm and used the location of ribs to estimate the volume of lungs in 400 images with various diseases. They performed a second part of the process after significant differences were found between estimated volumes and segmentation results. In this second part, characteristics based on texture were used to identify abnormal patterns in the image, which were then included in the result (Mansoor et al. 2017).

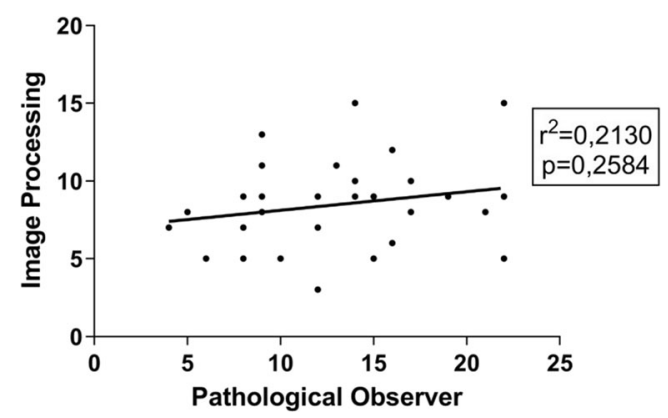

Fig. 5 In altered alveoli, correlation between analysis from the observing pathologist and computational analysis 
Wang et al. (2009) conducted a study with 31 volumes of normal cases and 45 volumes of abnormal cases. Before thresholding and applying texture extraction, they used an algorithm to label 3D-related components in the first 30 slices. They then identified the trachea location, which was used as a seed to trace the airways and prevent interference with lung segmentation.

To extract information from digital images, the images (and parts/objects that compose it) must be divided. This process is called image segmentation. It can be formally defined as an identification process (through a numerical algorithm) of the homogeneous regions of a specific image and the edges or limits of these regions. In most cases, these homogeneous regions are objects, and their edges are contoured (WANG et al. 2009).

Since the start of the COVID-19 pandemic, image processing of lungs has been frequently used to optimize diagnosis determination (as nucleic acid tests can result in falsenegatives for COVID-19) and monitor confirmed COVID19 cases (LI et al. 2021).

A systematic review demonstrated that digital processing of radiological images of the lungs to diagnose COVID-19 reduced false-positives and false-negatives (Ghaderzadeh and Asad 2021). Through the use of computed topography, machine learning has shown effectiveness in diagnosing other pulmonary complications and diseases, such as pulmonary emphysema (HUMPHRIES et al. 2020), fibrosis (CHRISTE et al. 2019), and pulmonary nodules (Nasrullah et al. 2019). These findings corroborate with the results of our study and show the effectiveness of digital image processing to diagnose lung lesions and pulmonary alterations, as well as justify the increasing use of digital image processing in clinical practice and scientific research.

The previously mentioned studies aimed to segment the lungs and lesions automatically by using a data set, without human interaction or a starting point of the anatomical location from other structures/previously defined models. But until now, no studies have used digital image processing with data and algorithms to examine the effect of herbicides on lung function.

The types of alterations caused by chronic 2,4-D inhalation exposure have not yet been defined; thus, it is not possible to obtain data for comparison. However, various studies have reported the occurrence of pulmonary alterations after acute and subacute oral exposure to 2,4-D. In one study, 2,4-D was added to the drinking water of rats. But rather than the lungs, this specific study investigated effects on the antioxidant defense system. It reported that herbicide ingestion led to a significant increase in the concentration of malondialdehyde (MDA) lipid peroxidation content (CELIK and TULUCE 2007).

By analyzing leukocytes from bronchialveolar lavage fluid, Kaur et al. (2021) reported lung inflammation in mice after oral exposure to high and low doses of 2,4-D for 90 days. The cytotoxicity of 2,4-D was analyzed in an in vitro study with human lung cells; the study reported an increase in apoptosistype cell death that was dependent on the herbicide dose. The study also reported that the induction of pulmonary cytotoxicity by 2,4-D occurs due to the rupture of the tubulinmicrotube cell network (Ganguli et al. 2014).

The human and rat respiratory systems are highly similar (FUSCO et al. 2002). Thus, this study can aid future research involving the analysis of histological images of human lung tissues to identify alveolar lesions through machine learning. However, to increase the amount of data for more accurate and effective image processing, studies must investigate the specific lung tissue lesions of herbicide-exposed populations.

Our study examined various types of histopathological alterations that characterize alveolar lesions, which made it difficult to identify a single pattern for image classification. In contrast, with healthy alveolar images, we recorded a significant correlation between pathologist and computational analyses. However, to obtain satisfactory image processing and diagnostic accuracy, a greater quantity of models and data volume is needed.

\section{Conclusion}

Computational analysis of the data revealed the existence of intact and healthy alveoli and damaged alveoli after herbicide 2,4-D exposure. This demonstrates the potential of digital data processing to improve medical diagnosis and research related to inflammation and mucus from chronic 2,4-D exposure. Few studies have used digital image processing methods in histopathological analysis. Further studies are necessary to understand immune system behavior after exposure to different doses of agricultural herbicides.

Author's contribution Claudio Pedrassoli Junior: lead author of the project, participated in the experiment, and wrote the paper

Gabriela Vidotto Cavallieri: participated in experiment design and production of histopathological slides

Francisco Assis da Silva: responsible for digital image processing

Geovana Letícia Fernandes: participated in experiment design and production of histopathological slides

Gisele Alborghetti Nai: participated in project design and writing of the paper

Ana Karina Marques Salge: participated in the analysis of the results

Josiano Guilherme Puhle: participated in the analysis of the results and review of the English language

Debora Tavares de Resende e Silva: participated in project design and analysis of results

Danillo Roberto Pereira: responsible for digital image processing

Fabíola de Azevedo Mello: participated in experiment design and production of histopathological slides

Ana Paula Alves Favareto: participated in project design, analysis of the results, and review of the paper 
Renata Calciolari Rossi: project coordinator, participated in experiment design, analysis of results, writing, and revision stages of the paper

Data availability All data generated or analyzed in this study are included in this published article (or its supplementary information files).

\section{Declarations}

Ethics approval and consent to participate Not applicable.

Consent for publication Not applicable.

Competing interests The authors declare no competing interests.

\section{References}

Abrasco. Brazilian Association of Public Health. Note against aerial spraying of insecticides for vector control (2016) Accessed on February 3, 2019. https: //www.abrasco.org,br/site/outrasnoticias/ institucional

Beaver RJ, Lehmann EL (2006) Nonparametrics: statistical methods based on ranks, 1ed edn. Springer-Verlag, New York

Celik I, Tuluce Y (2007) Determination of toxicity of subacute treatment of some plant growth regulators on rats. Environ Toxicol 22(6):613 619

Champely S. pwr: basic functions for power analysis. R package version. Disponível em: <https://cran.r-project.org/web/packages/pwr/pwr. pdf $>$. Acesso em: 31 ago. 2016

Christe A, Peters AA, Drakopoulos D, Heverhagen JT, Geiser T, Stathopoulou T, Christodoulidis S, Anthimopoulos M, Mougiakakou SG, Ebner L (2019) Computer-aided diagnosis of pulmonary fibrosis using deep learning and CT images. Investig Radiol 54(10):627-632

Committee for Update of the guide for the care and use of the laboratory animals: environment, housing and management. In: Guide for the care and use of laboratory animals. Washington: The National Academic Press, 2011. P. 41-103

Coppin B (2010) Artificial intelligence. LTC, Rio de Janeiro

de Mello F (2020) A. et al. Evaluation of genotoxicity after acute and chronic exposure to 2, 4-dichlorophenoxyacetic acid herbicide (2, 4D) in rodents using machine learning algorithms. J Toxicol Sci 45(12):737-750

de Mello F A, Quinallia G, Marion AC, Jorge FC, Marinelli LM, Salge AKM, de Fagiani M AB, Mareco EA, 2018 Favareto APA, Silva RCR e. Avaliação da cavidade nasal de camundongos submetidos à exposição ao herbicida ácido 2,4-diclorofenoxiacético. Medicina (Ribeirão Preto) [Internet]. 27 de dezembro de [citado 22 de abril de 2021]; 51(4):247-53. Disponível em: https://www.revistas.usp. $\mathrm{br} / \mathrm{rmrp} /$ article/view/154919.

Firetti R, Capanema LM, Fachini C, Turkish PHN, Son AAV (2012) Analysis of strategic variables for the development of agriculture in the Pontal do Paranapanema region. Econ Sociol Rural 50(1): 141-156 Brazilian

Fusco LB, Pêgo-Fernandes PM, Xavier AM, Pazetti R, Rivero DHRF, Capelozzi VL, Jatene FB (2002) Modelo experimental de enfisema pulmonar em ratos induzidos por papaína. J Pneumol 28(1):1-7

Ganguli A, Choudhury D, Chakrabarti G (2014) 2,4Dichlorophenoxyacetic acid induced toxicity in lung cells by disruption of the tubulin-microtubule network. Toxic Res 3(2):118-130
Ghaderzadeh M, Asad F (2021) Deep learning in the detection and diagnosis of COVID-19 using radiology modalities: a systematic review. J Healthc Eng 2021:6677314

Gomes MJM (2002) Ambiente e Lung. J Pneumol 28(5):261-269

Gonzalez RC, Woods RE (2010) Digital image processing, 3ed edn. Pearson, São Paulo

Humphries SM, Notary AM, Centeno JP, Strand MJ, Crapo JD, Silverman EK, Lynch DA (2020) Deep learning enables automatic classification of emphysema pattern at CT. Radiology 294(2):434 444

Kaur G et al (2021) Exposures to 2,4-Dichlorophenoxyacetic acid with or without endotoxin upregulate small cell lung cancer pathway. J Occup Med Toxicol 16(14)

Koerich A. (2012) Machine learningn.d.. Available at: http://www.ppgia. pucpr.br/ alekoe/AM/2012/0-Apresentacao-AM-2012.pdf. Accessed on April 22, 2019

Li T, Wei W, Cheng L, Zhao S, Xu C, Zhang X, Zeng Y, Gu J (2021) Computer-aided diagnosis of COVID-19 CT scans based on spatiotemporal information fusion. J Healthc Eng 2021:6649591

Mansoor A, Cerrolaza JJ, Perez G, Biggs E, Nino G, Linguraru MG (2017) Deep learning of the marginal form: applications to the segmentation of the pulmonary field in pediatrics. Proc SPIE Int Soc Opt Eng 10133:1013304. https://doi.org/10.1117/12.2254412

Nasrullah N, Sang J, Alam MS, Mateen M, Cai B, Hu H (2019) Automated lung nodule detection and classification using deep learning combined with multiple strategies. Sensors (Basel) 28;19(17):3722. https://doi.org/10.3390/s19173722

Otsu N (1979) A threshold selection method from gray-level histogram. IEEE Transaction on System Man Cybernetics 9(1):62-66

Parkinson CM, O'Brien A, Albers TM, Simon MA, Clifford CB, Pritchett-Corning KR (2011) Diagnostic necropsy and selected tissue and sample collection in rats and mice. J Vis Exp 54:2466

Pereira LM. Artificial intelligence myth and science. (2005). Available at: https://www.researchgate.net/profile/Luis_Pereira5/publication/ 237130636_Inteligencia_Artificial_Mito_e_Ciencia/links/ 00463527 ca46b52079000000/Inteligencia-Artificial-Mito-eCiencia.pdf?origin=publication_etigin. Accessed on April 22, 2019.

R Development Core Team (2019) R SoftwareR: a language and environment for statistical computing Vienna. http://www.r-project.org. Acessed 22 apr 2021.

Ruppert ADP, Pincelli MS, Souza HSP, Bigaton FJ, Soeiro ADM, Capelozzi VL, Serrano Jr CV (2012) Clinical manifestations and pulmonary histopathological analysis related to different diseases in patients with fatal pulmonary thromboembolism - an autopsy study. Rev Med (São Paulo) 91(2):69-82

Russ JC (1999) The image processing handbook, 3ed edn. CRC Press LLC., Raleigh, North Carolina

Sluimer IC, Van Waes PF, Viergever MA, Van Ginneken B (2003) Computer-aided diagnosis in high resolution CT of the lungs. Med Phys 30(12):3081-3090. https://doi.org/10.1118/1.1624771

Suzuki S, Abe K (1985) Topological structural analysis of digitized binary images by border following. Computer Vision, Graphics and Image Processing, CVGIP 30(1):32-46

Wang J, Li F, Li Q (2009) Automated segmentation of lungs with severe interstitial lung disease in CT. 36(Med Phys, 10):4592-4599. https://doi.org/10.1118/1.3222872

Publisher's note Springer Nature remains neutral with regard to jurisdictional claims in published maps and institutional affiliations. 